\title{
Validation of British Thoracic Society guidelines for the diagnosis of the sleep apnoea/hypopnoea syndrome: can polysomnography be avoided?
}

\author{
PJ Ryan, M F Hilton, D A R Boldy, A Evans, S Bradbury, S Sapiano, K Prowse, \\ R M Cayton
}

\begin{abstract}
Background-The British Thoracic Society report on the diagnosis and treatment of the sleep apnoea/hypopnoea syndrome (SAHS) suggests that, if the pulse oximetry baseline oxygen saturation is above $90 \%$, then $154 \%$ oxygen desaturations/hour in bed will diagnose SAHS requiring treatment. The diagnostic outcome of applying these guidelines has been studied.

Methods-One hundred patients referred to a district general hospital sleep clinic were recruited. After initial clinical assessment, overnight pulse oximetry measurements were performed, followed by full polysomnography at the regional laboratory.

Results-Sixty nine patients underwent both pulse oximetry and polysomnography. All 10 patients with more than 15 $4 \%$ desaturations/hour on pulse oximetry had SAHS confirmed on polysomnography (specificity $=100 \%$ ). Twenty two patients with SAHS were misdiagnosed using pulse oximetry alone (sensitivity= $31 \%$ ). These patients had low apnoea scores but high hypopnoea scores.

Conclusions-The BTS pulse oximetry criteria are highly specific when positive (specificity $=100 \%$ ), but may miss patients with the SAHS who have hypopnoeic episodes which cause arousal but not significant oxygen desaturation (sensitivity = $31 \%$ ). It should be emphasised that pulse oximetry alone does not always give sufficient information to discriminate between those patients with or without SAHS. Patients with "negative" pulse oximetry and symptoms of SAHS should undergo polysomnography or multichannel recording.

(Thorax 1995;50:972-975)
\end{abstract}

Keywords: sleep apnoea/hypopnoea syndrome, pulse oximetry, polysomnography.

Traditionally, polysomnography has been the method of choice for the diagnosis of the sleep apnoea/hypopnoea syndrome (SAHS). It is now apparent that this condition is far more prevalent than was previously thought and may affect $0 \cdot 3-9 \%$ of the population. ${ }^{1-3}$ Full polysomnography is expensive and time consuming, and the equipment and the expertise to run it is not widely available. In order to deal with the large numbers of patients now being referred to centres dealing with sleep-disordered breathing, and to facilitate easier diagnosis in nonspecialist hospitals, various other strategies to confirm or refute a diagnosis of SAHS have been evaluated..$^{4-8}$ These other methods have usually relied on pulse oximetry plus other criteria such as clinical features and symptom scores. ${ }^{458}$ The most widely used oximetry criteria for diagnosing the SAHS in the UK are those issued by a British Thoracic Society (BTS) working party in $1990 .^{9}$ These suggest that a diagnosis of the SAHS can be made if the awake baseline saturation is above $90 \%$ and there are at least $154 \%$ dips in oxygen saturation per hour in bed. The original guidelines suggested that oximetry studies in a district general hospital should take place in a quiet supervised room; however, the practice in our hospital and most others is for the patient to take an oximeter home to record overnight oximetry. It is in this context that it is now timely to assess these guidelines.

In this prospective study we have examined 100 consecutive patients referred to a sleep clinic in a district general hospital (DGH) for suspected SAHS using the BTS pulse oximetry criteria to make a diagnosis. These patients subsequently had full polysomnography at the regional sleep centre to establish whether the BTS guidelines were able to discriminate between patients with and without the SAHS.

\section{Methods}

The study population were referrals to the Birmingham Heartlands Hospital sleep clinic with suspected SAHS. Patients were excluded from the study if they were under 16 years of age, had an awake baseline oxygen saturation of $90 \%$ or less, or known cardiorespiratory, neuromuscular, or skeletal disease. The first 100 patients who satisfied these criteria then underwent clinical assessment including a patient and partner sleep questionnaire. Overnight home pulse oximetry arranged by Birmingham Heartlands Hospital (Minolta Pulsox-7) with a finger flexiprobe was then performed. This system samples every second but prints out the value at each 10 second interval (paper speed $=4 \mathrm{~mm} /$ minute). The oximetry trace was read by two respiratory physicians unaware of the clinical details. The number of desaturations was counted manually. The physicians were a consultant and a 
Table 1 Mean (SD) demographic data of 69 patients undergoing polysomnography

\begin{tabular}{lccc} 
& $\begin{array}{l}\text { Men } \\
(n=57)\end{array}$ & $\begin{array}{l}\text { Women } \\
(n=12)\end{array}$ & $\begin{array}{l}\text { Total } \\
(n=69)\end{array}$ \\
\hline Age (years) & $48(12)$ & $48(13)$ & $48(12)$ \\
Body mass index $\left(\mathrm{kg} / \mathrm{m}^{2}\right)$ & $29 \cdot 4(5 \cdot 0)$ & $30 \cdot 5(6 \cdot 3)$ & $29 \cdot 6(5 \cdot 2)$ \\
Systolic BP $(\mathrm{mm} \mathrm{Hg})$ & $143(19)$ & $140(20)$ & $142(19)$ \\
Diastolic BP $(\mathrm{mm} \mathrm{Hg})$ & $90(13)$ & $88(13)$ & $90(13)$ \\
\hline
\end{tabular}

senior registrar both with two to three years experience of running a respiratory sleep clinic. Using the BTS oximetry criteria, a diagnosis of "SAHS positive" or "SAHS negative" was made. Patients then underwent full polysomnography at the regional sleep laboratory at Stoke. This included oximetry (Ohmeda pulse oximeter 3700); respitrace recordings of abdominal and chest wall movements (Airshield impedance apnoea monitor); video recording of respiratory movements (Cannon vision $\mathrm{E}$ video camera with Akai recorder); two lead EEG, EMG, ECG and nasal and oral airflow measurements (Edentec airflow thermistor and PK Morgan Capnograph 901MK2) using the Neuroscience Sleepmaster system and software version X5.2D. Apnoeas were diagnosed on the basis of no airflow for at least 10 seconds and a desaturation of $>4 \%$ in the following 30 seconds. ${ }^{10}$ Hypopnoeas were defined as reduction in chest wall movement $(>25 \%)$, reduced abdominal wall movement $(>15 \%)$, and paradoxical movement with airflow reduction of $>25 \%$. The criteria of Rechtschaffen and Kales were used for sleep staging. ${ }^{11}$ These and respiratory events were computer analysed with manual editing. A subject was considered to have the SAHS if the apnoea/hypopnoea index was $>15 /$ hour of sleep judged on polysomnography alone.

Data for the study were analysed using Epi Info (version 5.0) statistical software and $p$ values were calculated using the Wilcoxon rank sum test.

\section{Results}

Of 100 patients entered into the trial 31 did not complete the protocol. Seven were discovered to have hypothyroidism on initial investigation, five had other medical conditions (three asthmatics, one myopathy, and one cardiac failure), seven had missing or incomplete data; 11 failed to attend all appointments, and one patient's overnight hypoxia was considered too severe for treatment to be delayed by awaiting polysomnography. Sixty nine patients (57 men) had both pulse oximetry at the hospital and polysomnography at the regional sleep laboratory. Demographic data are shown in table 1. The sources of referrals were: general practitioners $42 \%$, ENT surgeons $29 \%$, local chest physicians $19 \%$, other physicians $7 \%$. Interobserver agreement on the number of $4 \%$ desaturations was high $\left(r^{2}=0.96\right)$. Ten patients were diagnosed as having SAHS on pulse oximetry criteria alone. In all these patients subsequent polysomnography confirmed the pulse oximetry diagnosis of SAHS. Of the 59 patients whose oximetry was negative, 22 were found to have SAHS following polysomnography. The sensitivity of oximetry alone was $31 \%$ and the specificity was $100 \%$ (predictive value of a positive test $100 \%$, predictive value of negative test $63 \%$ ). Table 2 shows that there were a significantly greater number of apnoeas in the patients with SAHS diagnosed by oximetry (true positives) compared with polysomnography alone $(\mathrm{p}<0.05)$. However, the number of hypopnoeas in patients with SAHS, whether diagnosed by oximetry or not, was not statistically different. There was no obvious lower limit of oxygen desaturation frequency which excluded a diagnosis of SAHS with certainty. Comparison between the mean of the number of oxygen desaturations/hour for the false negative group compared with the true negatives was not statistically significant (mean 5.03 versus $3 \cdot 66$, respectively). A comparison of our results with those of other studies that have compared oximetry alone with polysomnography in patients referred for assessment of possible SAHS is given in table 3.

Nine of the 10 true positives were successfully established on nasal continuous positive airway pressure (NCPAP) (mean pressure $10 \mathrm{~cm} \mathrm{H}_{2} \mathrm{O}$ ) and one could not tolerate this treatment (table 2). Of the 22 false negative patients eight were successfully treated with NCPAP (mean pressure $7 \mathrm{~cm} \mathrm{H}_{2} \mathrm{O}$ ), two improved following uvulopalatopharyngoplasty, one required a mandibular prosthesis, and another improved with a weight reducing diet. Four patients were unwilling to use NCPAP and four were not considered to have symptoms severe enough to warrant treatment. Two patients repeatedly failed to attend the clinic and were discharged.

Outpatient pulse oximetry costs $£ 10$ per study when the cost of equipment, paper, technician time, and physician reporting time are taken into consideration. Inpatient polysomnography currently costs $£ 500$ per study when the same factors and the cost of a hospital bed are included. Screening 69 patients with home oximetry diagnosed 10 definite cases and cost only $£ 690$. Ten polysomnographies, which would have cost $£ 5000$, were avoided. Thus, a saving of $£ 4310$ ( $£ 62$ per patient) could have been made if oximetry was used to screen patients with suspected SAHS.

Table 2 Apnoealhypopnoea index and outcome in polysomnography (P) and oximetry (O) groups

\begin{tabular}{|c|c|c|c|c|c|c|c|}
\hline & $\begin{array}{l}\text { Diagnostic } \\
\text { group }\end{array}$ & Age & $\begin{array}{l}\text { BMI } \\
\left(\mathrm{kg} / \mathrm{m}^{2}\right)\end{array}$ & $\begin{array}{l}\text { Mean apnoea } \\
\text { index (range) }\end{array}$ & $\begin{array}{l}\text { Mean hypopnoea } \\
\text { index (range) }\end{array}$ & $\begin{array}{l}\text { Mean apnoeal } \\
\text { hypopnoea index (range) }\end{array}$ & $\begin{array}{l}\text { Successful NCPAP } \\
(\%)\end{array}$ \\
\hline $\begin{array}{l}\text { True negative } \\
\text { False negative } \\
\text { True positive }\end{array}$ & $\begin{array}{l}(\mathrm{O}-\mathrm{ve} / \mathrm{P}-\mathrm{ve}) \\
(\mathrm{O}-\mathrm{ve} / \mathbf{P}+\mathrm{ve}) \\
(\mathrm{O}+\mathrm{ve} / \mathrm{P}+\mathrm{ve})\end{array}$ & $\begin{array}{l}47 \\
51 \\
45\end{array}$ & $\begin{array}{l}28 \cdot 5 \\
28 \cdot 5 \\
35 \cdot 8\end{array}$ & $\begin{array}{c}5 \cdot 2(0-22) \\
15 \cdot 1(0-51) \\
\mathrm{p}<0.05\end{array}$ & $\begin{array}{c}32.9(4-123) \\
37.4(12-188) \\
p<0.5\end{array}$ & $\begin{aligned} 2 \cdot 7 & (0-14) \\
38 \cdot 1 & (15-123) \\
52 \cdot 5 & (15-90)\end{aligned}$ & $\begin{array}{l}15 \\
36 \\
90\end{array}$ \\
\hline
\end{tabular}

$\mathrm{BMI}=$ body mass index $\mathbf{N C P A P}=$ nasal continuous positive airway pressure . 
Table 3 Sensitivity and specificity of pulse oximetry compared with polysomnography in published series (in order of increasing specificity and decreasing sensitivity)

\begin{tabular}{|c|c|c|c|c|c|}
\hline Author & $n$ & Positive pulse oximetry criteria & Polysomnography criteria & Sensitivity & Specificity \\
\hline $\begin{array}{l}\text { Series } \\
(1993)^{12}\end{array}$ & 240 & $\begin{array}{l}\text { Qualitative assessment of } \mathrm{O}_{2} \\
\text { fluctuations by physician }\end{array}$ & $\begin{array}{l}\text { AHI }>10 \\
\text { AHI }>20\end{array}$ & $\begin{array}{l}98 \cdot 2 \\
100\end{array}$ & $\begin{array}{l}47 \cdot 7 \\
42 \cdot 6\end{array}$ \\
\hline $\begin{array}{l}\text { Rauscher } \\
(1993)^{13}\end{array}$ & 116 & $\begin{array}{l}\text { Cyclic oscillations of } \mathrm{O}_{2} \\
\text { and heart rate for }>30 \mathrm{~min}\end{array}$ & $\begin{array}{l}\mathrm{AHI}>10 \\
\mathrm{AHI}>20\end{array}$ & $\begin{array}{l}94 \\
95\end{array}$ & $\begin{array}{l}45 \\
41\end{array}$ \\
\hline $\begin{array}{l}\text { Cooper } \\
(1991)^{14}\end{array}$ & 46 & $\begin{array}{l}\text { Pattern recognition of } \\
\text { desaturations } \geqslant 5 \%\end{array}$ & $\begin{array}{l}\mathrm{AHI}>5 \\
\mathrm{AHI}>15 \\
\mathrm{AHI}>25\end{array}$ & $\begin{array}{r}60 \\
75 \\
100\end{array}$ & $\begin{array}{l}95 \\
86 \\
80\end{array}$ \\
\hline $\begin{array}{l}\text { Gyulay } \\
(1993)^{15}\end{array}$ & 98 & $\begin{array}{l}\geqslant 15 \text { desaturation } / \mathrm{h} \text { of } \\
\geqslant 2 \% \\
\geqslant 3 \% \\
\geqslant 4 \%\end{array}$ & $\mathrm{AHI}>15$ & $\begin{array}{l}65 \\
51 \\
40\end{array}$ & $\begin{array}{l}74 \\
90 \\
98\end{array}$ \\
\hline $\begin{array}{l}\text { Douglas } \\
(1992)^{16}\end{array}$ & 200 & $\begin{array}{l}\geqslant 4 \% \text { desaturations } \\
>5 / \mathrm{h} \\
>10 / \mathrm{h} \\
>15 / \mathrm{h} \\
>20 / \mathrm{h}\end{array}$ & AHI $>15$ & $\begin{array}{l}67 \\
53 \\
41 \\
36\end{array}$ & $\begin{array}{l}92 \\
97 \\
97 \\
99\end{array}$ \\
\hline $\begin{array}{l}\text { Williams } \\
(1991)^{4}\end{array}$ & 40 & $\begin{array}{l}\geqslant 4 \% \text { desaturations and } \\
\text { decreasing to } 90 \% \text { or less, } \\
\text { no frequency stated }\end{array}$ & $\mathrm{AI}>10$ & $65 \cdot 2$ & 100 \\
\hline $\begin{array}{l}\text { Bonsignore } \\
(1986)^{17}\end{array}$ & 83 & $\begin{array}{l}>204 \% \text { desaturations } / \mathrm{h} \text { of } \\
\text { duration }<0.9 \mathrm{~min}\end{array}$ & $\mathrm{AHI}>10$ & $74 \cdot 5$ & 100 \\
\hline $\begin{array}{l}\text { Current } \\
\text { study }\end{array}$ & 69 & $\begin{array}{l}>154 \% \text { desaturations } / \mathrm{h} \\
\text { (BTS guidelines) }\end{array}$ & $\mathrm{AHI}>15$ & 32 & 100 \\
\hline
\end{tabular}

$\mathrm{AHI}=$ apnoea/hypopnoea index; $\mathrm{AI}=$ apnoea index.

\section{Discussion}

As doctors and the general public have become more aware of the SAHS there has been a large increase in the numbers of new patients being referred for assessment. ${ }^{9}$ Polysomnography requires admission to a sleep laboratory, is time consuming, and the equipment and time required are expensive. Recently it has been demonstrated that sleep staging during polysomnography adds little to the overall clinical picture. ${ }^{16}$ Overnight polysomnography in a sleep laboratory has been regarded as the gold standard against which other methods of diagnosing the SAHS have been measured. ${ }^{18}$ One has to remember when comparing an investigation with polysomnography, that polysomnography itself is not "standard". It is argued that the standard by which a test must be judged is that which separates patients into groups that will or will not benefit from treatment. ${ }^{19}$ As polysomnography cannot always determine precisely which patients with a low or borderline apnoea/hypopnoea index require treatment, it cannot be wholly regarded as "golden" either.

The advent of home pulse oximetry offers an inexpensive method of screening for and diagnosing SAHS. It is less likely to disturb sleep and uses robust biological parameters of oxygen saturation and pulse rate. ${ }^{20}$ There are, however, a priori drawbacks with pulse oximetry. The clinically important event is arousal, whereas the pulse oximeter measures desaturations and changes in heart rate which are secondary phenomena. In practice, and notwithstanding these difficulties, pulse oximetry is widely used to screen for the SAHS and several studies have sought to compare its use with polysomnography. As can be seen from table 3, there are a number of difficulties in comparing these studies. There are wide variations in the criteria used to determine a positive oximetry trace and the number of apnoeas and hypopnoeas required to diagnose SAHS by polysomnography. Differences in patient populations and equipment compound those difficulties. One study in table 3 did not state the oximeter used, ${ }^{17}$ and the others used the Ohmeda 3700 which prints out the lowest value in each 12 second period. Our study used the Minolta Pulsox-7 which prints the measured oxygen saturation at 10 second intervals. Pulse oximetry is far from perfect and a low threshold for diagnosis will miss fewer patients with SAHS and give a high sensitivity at the expense of too many false positives and a low specificity. A higher oximetry threshold eliminates most false positives giving a high specificity but, as one would expect, the number of false negative results increases, resulting in a low sensitivity.

Our study shows how the BTS guidelines for the diagnosis of the SAHS are used with home oximetry by a district general hospital. It shows that, using the recommended criteria, there is high specificity but a large number of false negatives. Many of these patients in the false negative group were treated successfully with nasal CPAP. These findings are in close agreement with studies from tertiary referral centres when the same oximetry and polysomnography criteria are used. ${ }^{1314}$ We have also shown that the reason for the low sensitivity is that, whilst pulse oximetry is able to detect most episodes of apnoea, it tends to miss hypopnoeic episodes. This is because periods of hypopnoea, although causing clinically significant arousal and thus symptoms of daytime somnolence, are not always associated with falls in oxygen saturation. Of note is the lower body mass index in the false negative group compared with the true positive group. It has been suggested that a lower functional residual capacity in more obese patients may cause greater desaturation which may explain some of the difference in oximetry between the two groups. There is a danger that if one relies solely on pulse oximetry, many of the affected population will be missed. Untreated disease increases the risk of poor work performance, road traffic 
accidents, cardiorespiratory failure, stroke, and early death. ${ }^{19}$

How do these results translate into everyday clinical practice? The first point to note is that only just over two thirds of patients originally referred underwent both oximetry and polysomnography. There was a large number of non-attenders and incomplete data sets which we feel reflects both the difficulties for patients travelling 50 miles to the regional centre and the realities of NHS practice. Seven patients were found to have hypothyroidism at initial assessent and five had other medical conditions. This emphasises the need for full medical assessment at the initial consultation. The positive predictive value of pulse oximetry using the BTS criteria of $>154 \%$ oxygen desaturations per hour was $100 \%$ and, as such, obviates the need to perform polysomnography. In these cases treatment with NCPAP is started after appropriate investigations and lifestyle advice. The predictive value of a negative pulse oximetry test is only $63 \%$ - that is, over one third of patients in this group may have the SAHS. However, because of the low sensitivity of negative oximetry, many of these patients will require polysomnography to confirm or refute SAHS depending on the likelihood of SAHS before the test.

Can we do better using oximetry alone? By lowering the oximetry threshold we could expect to miss fewer patients with the SAHS, accepting a higher number of false positives. Douglas has shown that, even if only five $4 \%$ desaturations per hour are used to make a pulse oximetry diagnosis of SAHS, a number of true cases of SAHS are missed. ${ }^{16}$ Gyulay et al found that the cumulative time spent below $90 \%$ saturation was a more sensitive marker for sleep apnoea than counting repetitive desaturations, and that by combining the two measures oximetry was a better, though still imperfect, screening test. ${ }^{15}$

Combining pulse oximetry with other measurements seems to be the way forward if we wish to avoid performing full polysomnography in all patients. There has been some success in recording snoring sounds and matching these to reductions in oxygen saturation. Using a respiratory disturbance index of $>15$, sensitivity and specificity of $87 \%$ and $96 \%$ have been reported. ${ }^{6}$ Oximetry and respiratory movements using a static charge sensitive bed produced up to $100 \%$ sensitivity and $67 \%$ specificity, depending on the criteria evaluated. ${ }^{7}$

We have demonstrated the use of the BTS criteria for pulse oximetry for the diagnosis of SAHS in a district general hospital with a sleep clinic. By comparing the results with full polysomnography it is clear that positive pulse oximetry has a high predictive value and, in practical terms, is diagnostic of SAHS. However, there is a large group of patients with negative pulse oximetry, and these cases require further investigation if they have symptoms of daytime somnolence. The BTS guidelines are very useful when positive and may be used to avoid polysomnography and justify treatment. In the remaining group it should be made clear that $<154 \%$ desaturations/hour does not exclude significant SAHS. At the level of the district general hospital patients with $>154 \%$ desaturations/hour should be treated, while those with $<154 \%$ desaturations/hour and symptoms of daytime somnolence should be referred to a recognised sleep centre for polysomnography or multichannel recording if equipment and expertise is not available locally.

1 Stradling JR, Crosby JH. Predictors and prevalence of obstructive sleep apnoea in 1001 middle aged men. Thorax 1991;46:85-90.

2 McNamara SG, Grunstein RR, Sullivan CE. Obstructive sleep apnoea. Thorax 1993;48:754-64.

3 Young T, Palta M, Dempsey J, Skatrud J, Weber S, Badr $\mathrm{S}$. The occurrence of sleep disordered breathing among middle aged adults. $N$ Engl $\mathcal{F}$ Med 1993;328:1230-5.

4 Williams AJ, Yu G, Santiago S, Stein S. Screening for sleep apnoea using pulse oximetry and a clinical score. Chest 1991;100:631-5.

5 Viner S, Szalai JP, Hoffstein V. Are history and physical examination a good screening test for sleep apnoea? Ann Intern Med 1991;115:356-9.

6 Issa FG, Morrison D, Hadjuk E, Iyer A, Feroah T, Remmers JE. Digital monitoring of sleep disordered breathing using snoring sound and arterial oxygen saturation. Am Rev Respir Dis 1993;148:1023-9.

7 Svanborg E, Larsson H, Carlsson-Nordlander B, Pirskanen R. A limited diagnostic investigation for obstructive sleep apnoea syndrome. Chest 1990;98:1341-5.

8 Kapuniai LE, Andrew DJ, Crowell DH, Pearce JW. Identifying sleep apnoea from self-reports. Sleep 1988;11:430 6.

9 British Thoracic Society. Facilities for the diagnosis and treatment of abnormal breathing during sleep including nocturnal ventilation. BTS News 1990;5:7-10.

10 Guilleminault C, Partinen M. Obstrustive sleep apnoea syndrome. New York: Raven Press, 1990.

11 Rechtschaffen A, Kales A. A manual of standardised terminology, techniques and scoring for sleep stages in human subjects. Washington DC: National Institutes of Health, Publication No. 204, 1968.

12 Series F, Marc I, Cornier Y, La Forge J. Utility of noctural home oximetry for case finding in patients with suspected home oximetry for case finding in patients with suspected
sleep apnoea syndrome. Ann Intern Med 1933;119:449-53.

13 Rauscher H, Popp W, Zwick H. Model for investigating snorers with suspected sleep apnoea. Thorax 1993;48: 275-9.

14 Cooper BG, Veale D, Griffiths CJ, Gibson GJ. Value of nocturnal oxygen saturation as a screening test for sleep apnoea. Thorax 1991;46:586-8.

15 Gyulay S, Olsen LG, Hensley MJ, King MT, Murree AK, Saunders NA. A comparison of clinical assessment and home oximetry in the diagnosis of obstructive sleep home oximetry in the diagnosis of obstrir

16 Douglas NJ, Thomas S, Jan MH. Clinical value of polysomnography. Lancet 1992;339:347-50.

17 Bonsignore G, Marrone O, Macalso C, Salvaggio A, Stallone A, Bellia V. Validation of oximetry as a screening test for obstructive sleep apnoea syndrome. Eur Respir f 1990; 10(Suppl):542-4s.

18 American Thoracic Society. Indications and standards for cardiopulmonary sleep studies. Am Rev Respir Dis 1989; 139:559-68.

19 Stradling JR. Handbook of sleep-related breathing disorders. Oxford: Oxford University Press, 1993: 105-16.

20 Hutton P, Clutton-Brock T. The benefits and pitfalls of pulse oximetry. $B M F$ 1993;307:457-8.

21 Semple SJG, Gibson GJ. Sleep apnoea and related conditions. $\mathcal{F} R$ Coll Phys 1993;27:363-4. 